\title{
Comparative Physiological Pharmacokinetics of Fenatyl and Alfenatil in Rats and Humans Based on Parametric Single-Tissue Models
}

Sven Bjorkman, D. Russell Wada, Donald R. Stanski, and William F. Ebling

This footnote to Table II was originally to appear in Journal of Pharmacokinetics and Biopharmaceutics, Vol. 22, No. 5, on page 387.

${ }^{\mathrm{a}}$ The C.V. (\%) of the fitted value is given within parenthesis. In the whole-body models, the carcass was characterized using the parameters for skin. See Glossary for explanation of the parameters. 